\title{
Biosynthesis of Gold Nanoparticles by Fenugreek (Trigonella Foenum-Graecum) Extract
}

\author{
Ahmed Fragoon*,1, Lamiaa Frah², Amal Mamoun ${ }^{2}$ \\ ${ }^{1}$ PHD, Department of Biomedical Engineering, Sudan University of Science and Technology, 11111, Sudan \\ ${ }^{2}$ BSC, Department of Biomedical Engineering, Sudan University of Science and Technology, 11111, Sudan
}

\begin{tabular}{l} 
A R T I C L E I N F O \\
\hline Article history: \\
Received: 30 September, 2016 \\
Accepted: 14 October, 2016 \\
Online: 27 October, 2016 \\
\hline Keywords: \\
Biosynthesis \\
fenugreek seed extract \\
antioxidant \\
Biocompatibility \\
\hline
\end{tabular}

\begin{tabular}{l} 
A B S T R A C T \\
\hline In this work fenugreek(Trigonella Foenum Graecum) extract was used to synthesis gold \\
nanoparticles by simple and safe method .In aqueous solution of chloroaurate ions, \\
fenugreek extract was used as reducing agent and gum Arabic as stabilizer by one synthetic \\
route which is microwave irradiation.The nanoparticles were characterized and \\
investigated by ultraviolet-visible (UV-Vis) spectrophotometry, transmission electron \\
microscopy (TEM), energy-dispersive X-ray (EDX) spectroscopy, X-ray diffraction \\
(XRD)and Fourier transform infrared spectroscopy(FTIR). The size and shape of the \\
nanoparticles were found to be very sensitive to the quantity of the extract. And the shape \\
of gold nanoparticles was found to be multiform; the EDX spectrum show high peaks of \\
gold that indicate the pure nature of gold nanoparticles in chemical composition. \\
\hline
\end{tabular}

\section{Introduction}

This paper is an extension of work originally presented in 2015 International Conference on Computing, Control, Networking, Electronics and Embedded Systems Engineering (ICCNEEE) [1]. Nanotechnology, nanoscience, nanostructure, nanoparticles are now of the most widely used words in scientific literature. Nanoscale materials are very attractive for possible machine, which will be able to travel through the human body and repair damaged tissues or supercomputers which small enough to fit in shirt pocket. However, nanostructure materials have potentials application in many other areas, such as biological detection, controlled drug delivery, low-threshold laser, optical filters, and also sensors, among others [2-3].

In fact, metal nanoparticles have been used a long time ago e.g. Damascus steel which used to make sword and Glass Lycurgus Cup which has unique color. Even though, nanoparticles have been used a long time ago, but nobody realized that it reached nanoparticles scale[4-7], One of the most interesting aspects of metal nanoparticles is that their optical properties depend strongly upon the particle size and shape ,Bulk Au looks yellowish in

\footnotetext{
${ }^{*}$ Corresponding Author: Ahmed Fragoon, Khartoum, Sudan, +249991784173 ,

lamiaafarh1992@gmail.com, ahmedfragoon@sustech.edu
}

reflected light, but thin Au films look blue in transmission. This characteristic blue color steadily changes to orange, through several tones of purple and red, as the particle size is reduced down to $\sim 3 \mathrm{~nm}$. These effects are the result of changes in the socalled surface Plasmon resonance [8], the frequency at which conduction electrons oscillate in response to the alternating electric field of incident electromagnetic radiation. However, only metals with free electrons (essentially $\mathrm{Au}, \mathrm{Ag}, \mathrm{Cu}$, and the alkali metals) possess Plasmon resonances in the visible spectrum, which give rise to such intense colors. Elongated nanoparticles (ellipsoids and nanorods) display two distinct Plasmon bands related to transverse and longitudinal electron oscillations. The longitudinal oscillation is very sensitive to the aspect ratio of the particles [9-10].

Most commonly studied metal nanoparticles include gold, silver, titanium oxide and iron nanoparticles. Among these, gold being inert and relatively less cytotoxic is extensively used for various applications [11].

Gold nanoparticle chemistry and physics has emerged as a broad new subdiscipline in the domain of colloids and surfaces. The unusual optical properties of small gold particles, their size dependent electrochemistry, and their high chemical stability 


\section{A. Fragoon et al. / Advances in Science, Technology and Engineering Systems Journal Vol. 1, No. 5, 50-55 (2016)}

have made them the model system of choice for exploring a wide range of phenomena including self-assembly, biolabeling, catalysis, electron-transfer theories, phase transfer, DNA melting and assays, and crystal growth [12].

Among the use of living organisms for nanoparticle synthesis, plants have found application particularly in metal nanoparticle synthesis. Use of plants for synthesis of nanoparticles could be advantageous over other environmentally benign biological processes as this eliminates the elaborate process of maintaining cell cultures. Biosynthetic processes for nanoparticles would be more useful if nanoparticles were produced extracellularly using plants or their extracts and in a controlled manner according to their size, dispersity and shape. Plant use can also be suitably scaled up for large-scale synthesis of nanoparticles [13].

Fenugreek (Trigonella foenum-graecum) belongs to the family fabaceae. Fenugreek is used both as a herb (the leaves) an as a spice (the seed), has been used for long time as a hematopoietic and antioxidant agent in traditional therapeutics, in Sudan the seeds have many uses especially in folk medicine. Whole seeds are swallowed as antiacid and against dysentery and stomach disturbances [14-15].

Fenugreek have been shown it contain high levels of antioxidants in which may be due partly to the presence of flavonoids and polyphenols. In this paper, we present a detailed study of the biosynthesis of GNPs by the reaction of fenugreek extract with aqueous gold ions. We hypothesized that the effective utilization the various phytochemicals present in fenugreek and their antioxidant activities will provide simple, clean, nontoxic and environmentally being bioreduction process of gold salts into their corresponding nanoparticles.In additional, the obtained GNPs were further stabilized by gum Arabic, another plant source glycol protein[16].

The emphasis is on controlling the size and shape of the GNPs by varying the experimental conditions in the synthesis and thereby modulates the optical properties of the nanoparticles. Up to now, many efforts have been made to fabricate anisotropic gold nanostructures with various shapes [17]. Although some chemicals methods for the preparation of GNPs with pre-chosen size by changing the concentration of gold ions and stabilizer [18], or by seed mediated growth [19], they may utilize toxic chemicals either in the form of reducing agents or as stabilizing agents. However, a few works have reported on the biosynthesis of nanostructures with novel and controllable size or desirable shape. Here, we show that size control can be achieved by simple variation in the quantity of the fenugreek extract in the reaction medium. In this process, we use a household microwave oven as a heating apparatus to synthesize gold colloids. Microwave chemistry, which reviewed in detail in the areas of organic reactions [19] and analytical chemistry [20], synthesized nanoparticles were characterized by various methods, such as transmission electron microscopy (TEM), energy-dispersive Xray $(\mathrm{EDX})$, ultraviolet-visible $(\mathrm{UV}-\mathrm{Vis})$ and $\mathrm{X}$-ray diffraction (XRD) [21].

\section{Experimental Details}

\subsection{Materials}

The fenugreek seed and the gum arabic powder were purchased from a local herbal shop in the Sudan. Hydrogen tetrachloroaurate tetrahydrate $\left(\mathrm{HAuCl}_{4} .3 \mathrm{H}_{2} \mathrm{O}\right)$ purchased from Labline Co. Ltd. (Sudan) and used without further purification.

\subsection{Instrumentations}

\subsubsection{UV-Vis Absorption Spectroscopy}

Optical absorption spectra of the fenugreek seed ,extract reduced GNPs were recorded using a UV-1800 UV-Visspectrophotometer (Shimadzu, Japan) with $2 \mathrm{ml}$ of GNPs solution in a $1 \mathrm{~cm}$ optical path cuvette.

\subsubsection{Transmission Electron Microscopy}

The morphology and size of the GNPs were analyzed using the TEM images obtained with transmission electron microscope JEM 2100200 kV (JEOL, Japan).

\subsubsection{Energy Dispersive X-Ray Spectroscopy}

The chemical composition and element composition maps of GNPs were analyzed using EDX plot obtained with Energy Dispersive X-Ray Spectroscopy.

\subsubsection{X-ray Powder Diffraction}

The phase identification and crystal structures of GNPs were characterized using the XRD plot obtained with X-ray Powder Diffraction labx XRD 6000(Shimadzu, Japan). The GNPs solution were converted to dry by centrifuged $(6000 \mathrm{rpm}$ for 50 min) and dried under sun.

\subsubsection{Fourier transforms infrared spectroscopy}

The infrared spectrum of absorption, emission, photoconductivity or Raman scattering of GNPs were analyzed using FTIR plot obtained with Fourier transform infrared spectroscopy FTIR-8400S (Shimadzu, Japan).

\subsection{Preparation of fenugreek Seeds Extract}

Weighted $8 \mathrm{~g}$ from fenugreek seed then washed with sterilized water to remove any contaminant or dust particles, putted on filter paper to dry the beaker washed with sterilized water and sterilized in oven at $200^{\circ} \mathrm{C}$ for $30 \mathrm{~min}$. The fenugreek seed putted in $50 \mathrm{ml}$ dionizd water at beaker and covered with plastic cover. Then it was incubate for $24 \mathrm{~h}$ at room temperature.

After the incubation period the solution of the fenugreek was centrifuged at $6000 \mathrm{rpm}$ for $15 \mathrm{~min}$, than it was stored at $4^{\circ} \mathrm{C}$, and use within 3 days for GNPs synthesis.

\subsection{Biosynthesis of GNPs by Microwave Irradiation}

In a typical experiment, to $200 \mathrm{ml}$ beaker was added $120 \mathrm{mg}$ of gum arabic powder, $10 \mathrm{ml}$ of fenugreek seed extract and the volume increased to $20 \mathrm{ml}$ by addition of an appropriate volume of deionised water. To the resulting mixture $28 \mathrm{ml}$ aqueous solution of $10 \mathrm{mM}\left[\mathrm{HAuCl}_{4} \cdot 3 \mathrm{H}_{2} \mathrm{O}\right]$ was immediately added. 
Following this, the beaker was placed in the centre of a domestic microwave oven (MS3040S/00) at 2450MHZ. After just $30 \mathrm{~s}$ of microwave irradiation at the maximum power output of $850 \mathrm{~W}$, the color of the stirred mixture turned purple-red from pale yellow indicating the formation of GNPs. The solution was then left to cool to room temperature and the rapid reduction is complete within $2 \mathrm{~min}$ as shown by stable light purple- red color of the solution which gives $10 \mathrm{ml}$ colloid. To obtain 8 and $6 \mathrm{ml}$ colloids the addition of the fenugreek seed extract is varied as $8,6 \mathrm{ml}$, respectively.

\section{Result and Discussion}

In the present study, Gold nanoparticles exhibit a distinct optical feature commonly referred to as localized surface Plasmon resonance (LSPR), that is, the collective oscillation of electrons in the conduction band of gold nanoparticles in resonance with a specific wavelength of incident light. LSPR of gold nanoparticles results in a strong absorbance band in the visible region $(500 \mathrm{~nm}-$ $600 \mathrm{~nm}$ ), which measured by UV-Vis spectroscopy .The LSPR spectrum is dependent both on the size, and shape of gold nanoparticles. The peak absorbance wavelength increases with particle diameter, and for uneven shaped particles such as gold nanourchins, the absorbance spectrum shifts significantly into the far-red region of the spectrum when compared to a spherical particle of the same diameter .Figure 1 below show UV-VIS curves for absorption of synthesized GNPS, the absorption at the wavelength range from $400 \mathrm{~nm}-700 \mathrm{~nm}$.

\subsection{UV-VIS Result}

The template is used to format your paper and style the text. All margins, column widths, line spaces, and text fonts are prescribed; please do not alter them. You may note peculiarities. For example, the head margin in this template measures proportionately more than is customary. This measurement and others are deliberate, using specifications that anticipate your paper as one part of the entire proceedings, and not as an independent document. Please do not revise any of the current designations.

\subsection{TEM Result}

The morphology of GNPs was characterizing using TEM images recorded at different magnifications of gold particles resulting from the reduction of chloroaurate ions by different amounts of the fenugreek extract. Figure2 (A),(B),(C) and (D) show GNPs reduce by $8 \mathrm{ml}$ fenugreek extract ,its consist of branched (multipods) hexagonal with spherical, triangular, pentagon, and rod nanoparticles .when $10 \mathrm{ml}$ fenugreek extract was used to reduce the aqueous $\mathrm{HAuCl} 4$, figure 3(A),(B),(C) and (D ) consist of branched (multipods) spherical with triangular, rod, and hexagonal nanoparticles .According to the size distribution of the spherical GNPs shown in figure 4 (A) and (B) the nanoparticles, the average size ca $13.71 \mathrm{~nm}$ for $8 \mathrm{ml}$ FSE and average of size ca. $11.85 \mathrm{~nm}$ for $10 \mathrm{ml} \mathrm{FSE}$.

In conclusion, Biosynthesis of GNPs using fenugreek extract produce multiform GNPs.

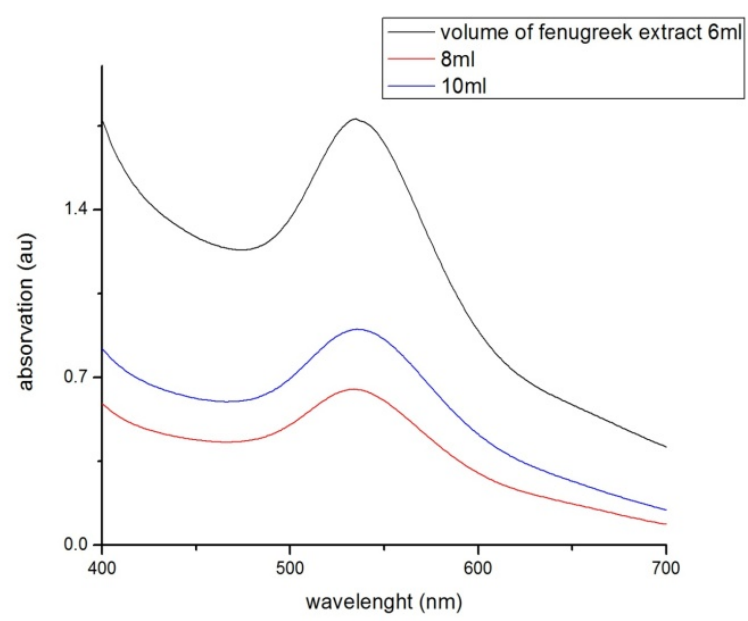

Figure 1 Absorption spectra of GNPs after bioreduction by fenugreek Extract of 6,8 and $10 \mathrm{ml}$ dosages were exposed to $28 \mathrm{ml}, 10 \mathrm{mM}$ Aqueous solution of $\mathrm{HAuCl}_{4}$.
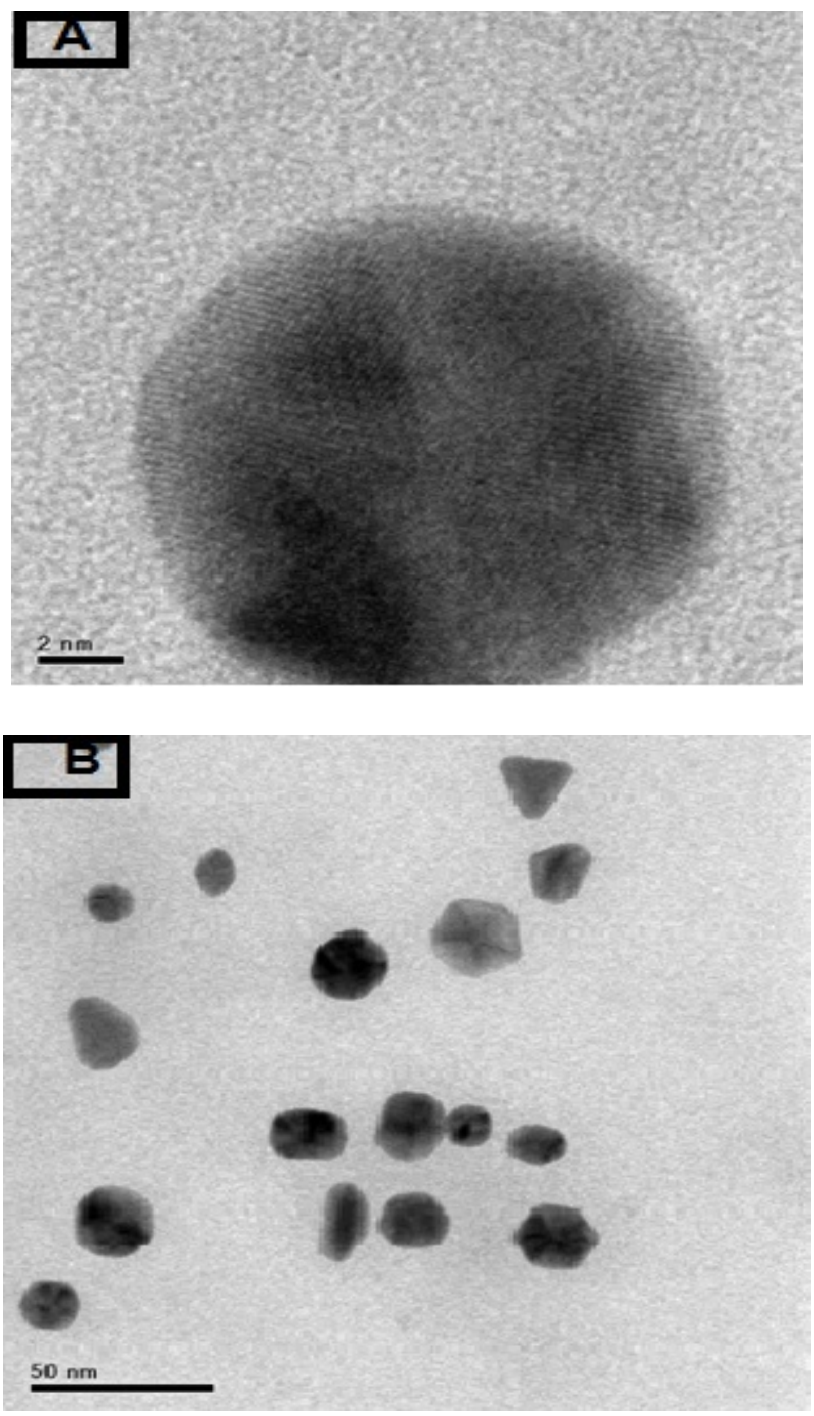


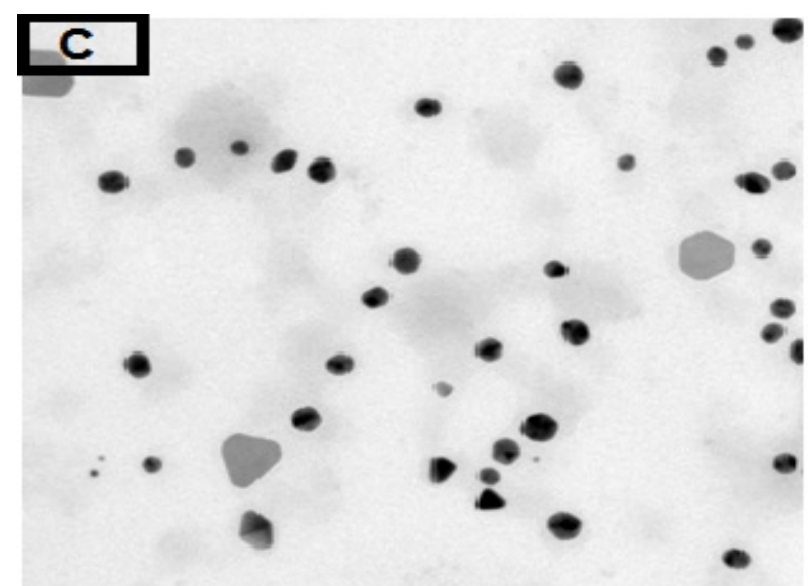

\section{$900 \mathrm{~nm}$}

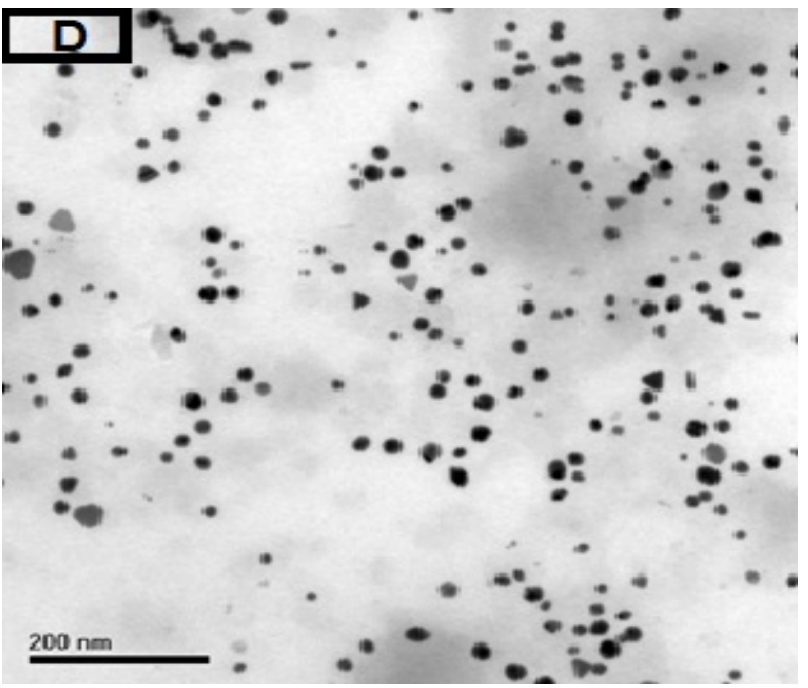

Figure 2 TEM images illustrating the biosynthesis of GNPs using microwave irradiation by exposing $8 \mathrm{ml} \mathrm{FSE} \mathrm{to} 28 \mathrm{ml}, 10 \mathrm{mM}$ aqueous $\mathrm{HAuCl}_{4}$, Scale bars (A) $2 \mathrm{~nm}$,(B) $50 \mathrm{~nm}$ (C) $100 \mathrm{~nm}$ and (D) $200 \mathrm{~nm}$.
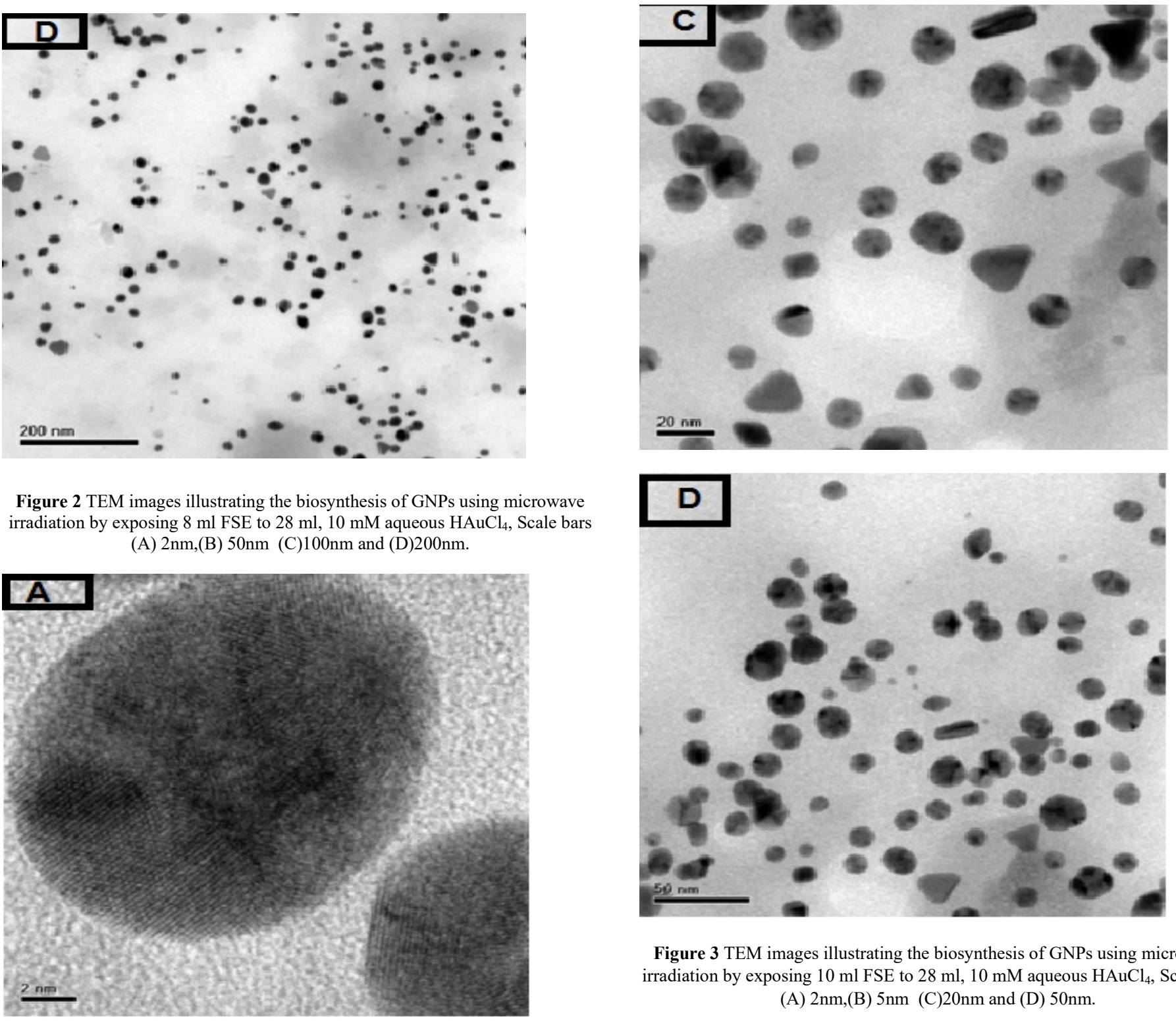

Figure 3 TEM images illustrating the biosynthesis of GNPs using microwave irradiation by exposing $10 \mathrm{ml} \mathrm{FSE}$ to $28 \mathrm{ml}, 10 \mathrm{mM}$ aqueous $\mathrm{HAuCl}_{4}$, Scale bars (A) $2 \mathrm{~nm}$,(B) $5 \mathrm{~nm}$ (C) $20 \mathrm{~nm}$ and (D) $50 \mathrm{~nm}$. 


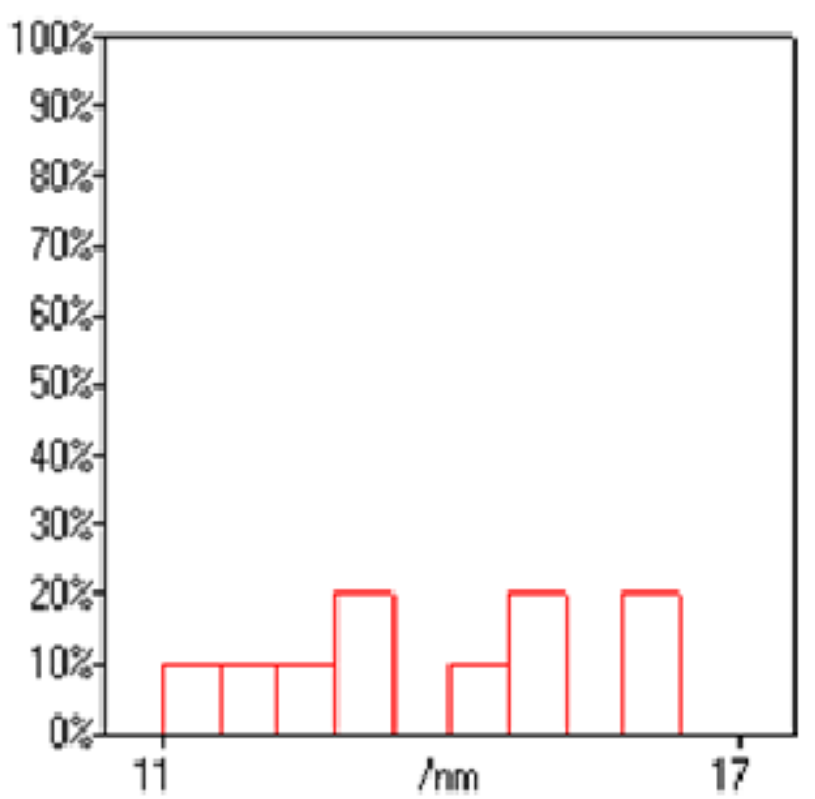

(A)

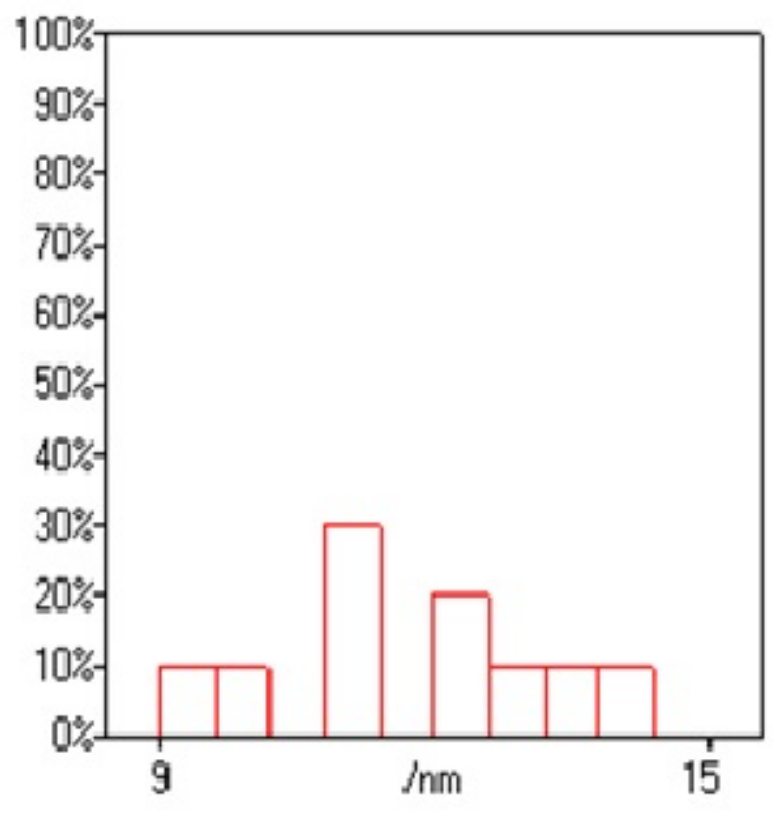

(B)

Figure 4 A histogram of size distribution of spherical GNPs synthesized using (A) $8 \mathrm{ml} \mathrm{FSE} \mathrm{(B)} 10 \mathrm{ml} \mathrm{FSE}$.

\subsection{EDX Result}

In the EDX spectrum of the GNPs, Figure5 (A) showed that a typical biogenic nanoparticle exhibited strong X-ray emission signals from the gold atoms and the $\mathrm{Cu}$ peaks were due to background signals from the supporting grid. This indicates that the biogenic GNPs are relatively pure in chemical composition.

XRD analysis figure5(B) show Three peaks observed at $38.3^{\circ}$, $40.6^{\circ}$, and $50.4^{\circ}$, can be indexed to the $(111),(200)$, and $(220)$ reflections of face-centered cubic (FCC) structure of metallic $\mathrm{Au}$, respectively, showing the pure crystalline nature of the prepared www.astesi.com particles. The intensity ratio between the (200) and the (111) diffractions $(\mathrm{I}(200) / \mathrm{I}(111))$ of 1.43 for the prepared sample is larger than the conventional bulk intensity ratio $(\sim 0.53)$.

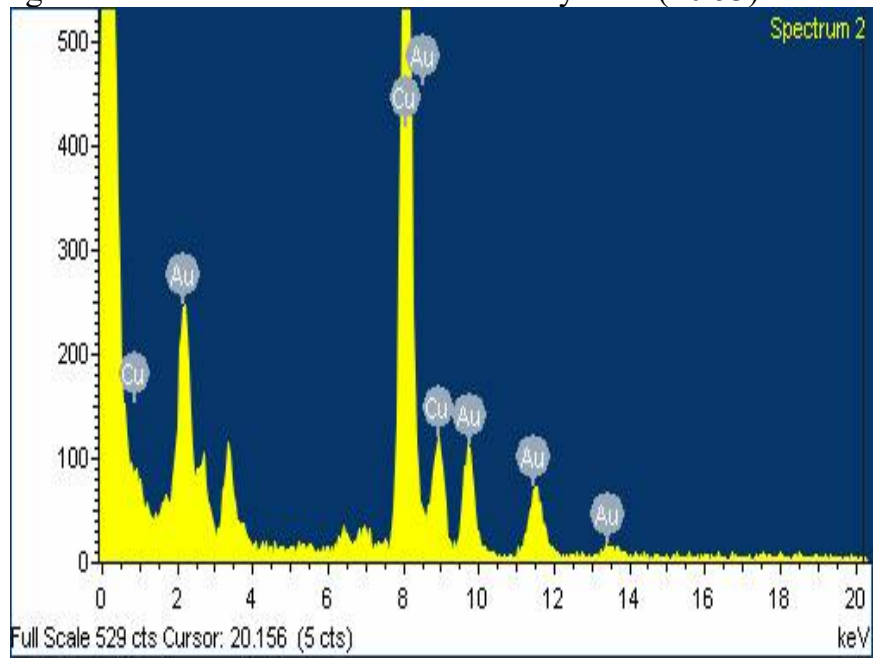

(A)

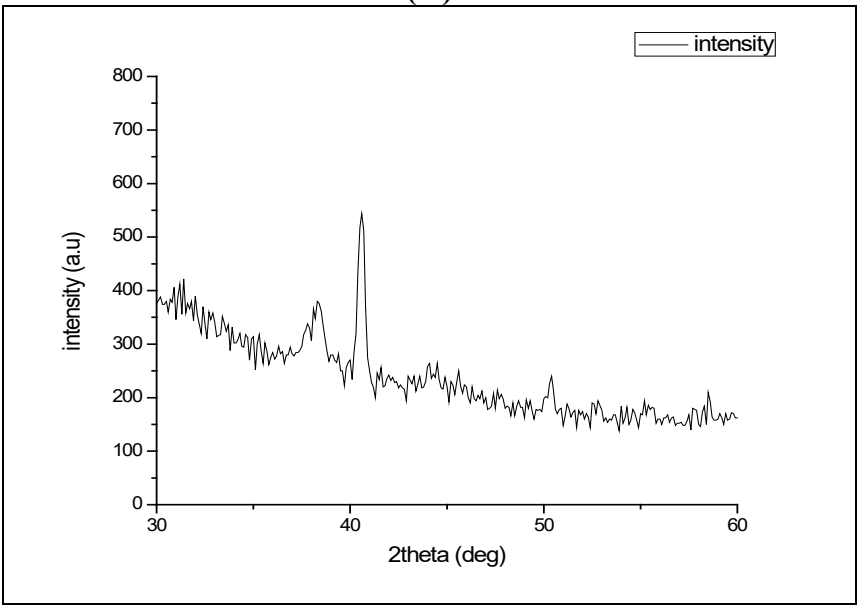

(B)

Figure 5 (A) EDX spectrum of GNPs from the inset image, (B) X-ray diffraction patterns of $8 \mathrm{ml} \mathrm{FSE}$ with $28 \mathrm{ml}, 10 \mathrm{mM}$ aqueous $\mathrm{HAuCl}_{4}$.

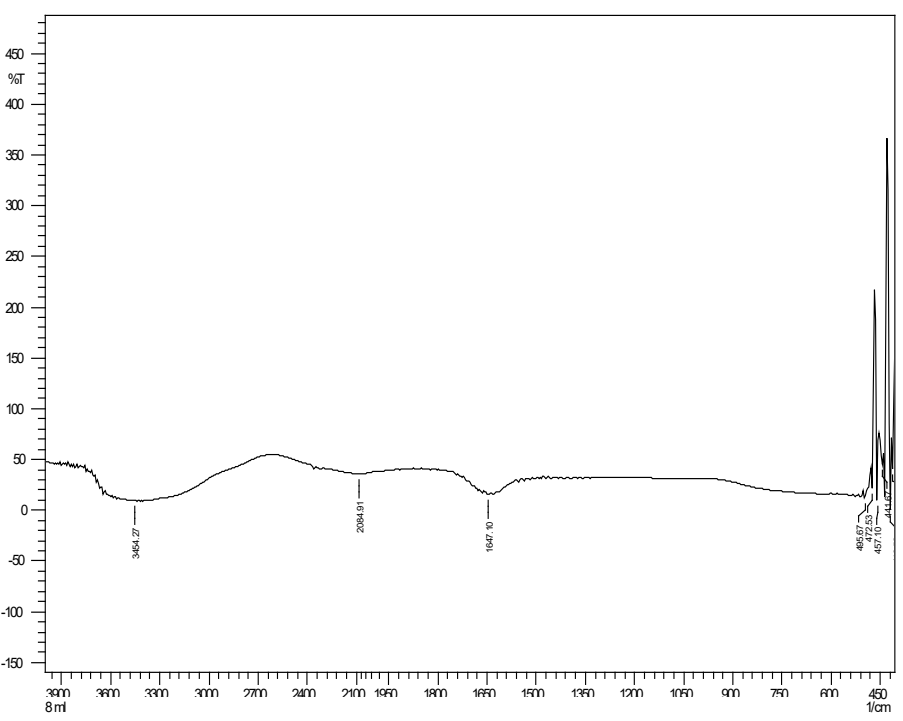

Figure 6 FTIR spectrum of FSE with $28 \mathrm{ml}, 10 \mathrm{mM}$ aqueous $\mathrm{HAuCl}_{4}$. 


\subsection{Fourier transform infrared spectrum Result}

Fourier transform infrared spectrum show the active components that have been detected Phenols and Alkaloid. The extracts of the fenugreek exhibit antioxidant activity, chemical composition and polar nature of FSE contains phenol compound. IR spectrum show peaks at 3454.27, 2084.91, 1647.10, 495.67 $\mathrm{cm}-1$ correspond to $(\mathrm{O}-\mathrm{H}$ stretch, $\mathrm{H}$-bonded $)$ phenols, $(-\mathrm{C} \equiv \mathrm{C}-)$ stretch/ alkynes, $(-\mathrm{C}=\mathrm{C}-)$ stretch/ alkenes. As figure 6 shows.

\section{Conclusion}

The work defines the synthesis of GNPs in aqueous solution of chloroaurate ions using fenugreek extract as reducing agent and gum arabic as stabilizer. This method produced biocompatible GNPs simply. One synthetic route, microwave irradiation his been used. The study finds that biosynthesis of GNPs using fenugreek extract produce multiform and uncontrollable gold nanoparticle. The study finds that as the amount of fenugreek extract increased the size diameter of GNPs increase. In further studies, these functional GNPs could be used in different medical application like vaccine storage indicator methods.

\section{Acknowledgment}

This work was supported by Sudan University of Science and Technology department of Biomedical Engineering, research lap, department of Medical Laboratory Sciences, Africa Technology City and University of Medical Sciences.

\section{References}

[1] Ahamed Fragoon, Amal Mamoun, Lamiaa Frah, Shahinaz Abd Alwahab (biosynthesis of Gold Nanoparticle by Fenugreek Seed (Trigonella Foenum Extract) (2016).

[2] Kostoff, R. N.; Koytcheff, R. G.; Lau, C. G. Y. (Structure of the nanoscience and nanotechnology applications literature). The Journal of Technology Transfer, 1-13

[3] Andrievskii, R. A. Directions in Current Nanoparticle Research. Powder Metallurgy and Metal Ceramics, 42 (11): 624-629 (2003).

[4] Liz-Marzan, L. M. (Nanometals: Formation and color). Materials Today, 7(2): 26-31 (2004)

[5] Roco, M. C. (Nanoparticles and Nanotechnology Research) Journal of Nanoparticle Research, 1(1): 1-6 (1999).

[6] Reibold, M.; Paufler, P.; Levin, A. A.; Kochmann, W.; Patzke, N.; Meyer, D. C. Materials: Carbon nanotubes in an ancient Damascus sabre. Nature 2006.

[7] Fredy Kurniawan (new analytical application of gold nanoparticale) Surabaya, Indonesia march 2008

[8] U. Kreibig, M. Vollmer (Optical Properties of Metal Clusters), SpringerVerlag, Berli.

[9] S. Link, M.A. El-Sayed J. Phys. Chem. B, 103: 8410 (1999).

[10] Luis M Liz_Marzan (Nanometals: Formation and color) materialstoday 23 January 2004.

[11] Pooja M. Tiwari, Komal Vig, Vida A. Dennis and Shree R. Singh (Functionalized Gold Nanoparticles and Their Biomedical Applications) Nanomaterials, 1: 31-63 (2011).

[12] Rajesh Sardar, Alison M. Funston, Paul Mulvaney, and Royce W. Murray (Gold Nanoparticles: Past, Present, and Future) Langmuir, 25(24): 1384013851 (2009).

[13] V. Kumar and S. K. Yadav, J. Chem (Plant-mediated synthesis of silver and gold nanoparticles and their applications) 84: 151 (2009).
[14] Abdel Moneim E. Sulieman1, Heba E. Ahmed2and Awad M. Abdelrahim( The Chemical Composition of Fenugreek (Trigonella Foenum graceum L) and the Antimicrobial Properties of its Seed Oil) Wad-Medani, Sudan 3(2).

[15] Ghosh, Sourav; Sengupta, Jayeeta; Datta, Poulami; Gomes, Antony (Hematopoietic and Antioxidant Activities of Gold Nanoparticles Synthesized by Aqueous Extract of Fenugreek (Trigonella foenum-graecum) Seed), 546-5527 (2014).

[16] L. Huang, M. Wang, Y. Zhang, Z. R. Guo, J. F. Sun, and N. J. Gu,Phys. Chem. C 111: 16154 (2007).

[17] D. V. Leff, P. C. Ohara, J. R. Health, and W. M. Gelbart, J. Phys.Chem. 99: 7036 (1995).

[18] T. K.Sau, A. Pal, N. R. Jana, N. L. Wang, and T. Pal, J. Nanopart.Res. 3: 257 (2001).

[19] R. N. Gedye, W. Rank, and K. C. Westaway, Can. J. Chem. 69: 706 (1991).

[20] J. R. J. PareÂ, J. M. R. BeÂlanger, and S. S. Stafford, Trends Anal.Chem. 13: 176 (1994).

[21] Fragoon, J.Li, J.Zhu, and J. Zhao (Biosynthesis of Controllable Size and Shape Gold Nanoparticles by Black Seed (Nigella Sativa) Extract) (2012). 